\title{
Mailbox \\ Cover-preserving embeddings of finite length semimodular lattices into simple semimodular lattices
}

\author{
E. TAmás Schmidt
}

Abstract. We prove that every semimodular lattice of finite length has a cover-preserving embedding as a filter into a simple semimodular lattice.

G. Grätzer and E. W. Kiss [3] proved the following deep theorem: every finite semimodular lattice $L$ has a cover-preserving embedding into a finite simple geometric lattice. For semimodular lattices of finite length see G. Czédli and E. T. Schmidt [1]. By the length, $\ell(L)$, of a lattice $L$ we mean the number $\sup \{n \mid L$ has an $(n+1)$ - element chain $\}$.

If we drop 'geometric', G. Grätzer and T. Wares [5] proved that every finite semimodular lattice has a cover-preserving embedding into a simple semimodular lattice. Their proof is relatively short and uses the one-element extension introduced in [4]. In the present note we give a very short proof of this result.

Theorem. Every semimodular lattice $L$ of finite length has an embedding $\varphi$ into a simple semimodular lattice $\bar{L}$ which satisfies the following conditions:

(1) $\varphi$ is cover-preserving,

(2) $L \varphi$ is a filter of $\bar{L}$,

(3) $\ell(\bar{L})=\ell(L)+1$ and $|\bar{L}|=|L|+2 \ell(L)+1$.

Proof. Let $L$ be a semimodular lattice of finite length. Take a maximal chain $C$ of $L$. Consider $\{0,1\} \times C$ and add new elements to the covering squares making them into copies of the lattice $M_{3}$. We get a lattice $K$ that contains a filter $C^{\prime}=\{(1, c) ; c \in C\}$ isomorphic to $C$ under the isomorphism $\psi: C \rightarrow C^{\prime}$, which sends every $c \in C$ to $(1, c)$. Consider the attachment $\bar{L}$ of the lattice $K$ to the lattice $L$ over $C$ by identifying $C$ with $C^{\prime}$ along $\psi$, in the sense of G. Grätzer and D. Kelly [2] (see the Figure). So we have $\bar{L}=L \cup K$ with $L \cap K=C$ and the order

$$
a \leq b= \begin{cases}a \leq_{K} b & \text { if } a, b \in K ; \\ a \leq_{L} b & \text { if } a, b \in L ; \\ a \leq_{K} x \psi, x \leq_{L} b & \text { if } a \in K, b \in L, \text { for some } x \in C .\end{cases}
$$

The poset $\bar{L}$ is obviously a lattice and $L$ is a filter, which proves (2). To show that $\bar{L}$ is semimodular, we have to verify the condition: $a \prec b$ implies $a \vee d \preceq b \vee d$.

Key words and phrases: Lattice, semimodular, finite length, simple, embedding.

This research was supported by the Hungarian National Foundation for Scientific Research (OTKA), grant no. K 77432 . 


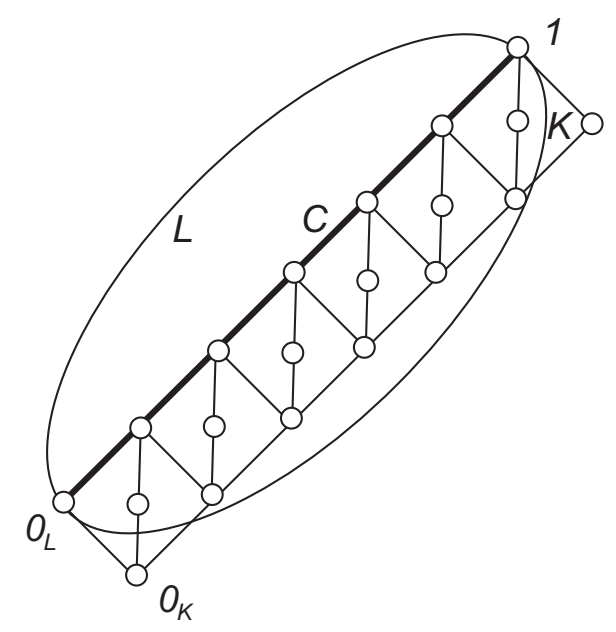

There is only one nontrivial case: $a \in \bar{L}-L, d \in \bar{L}-K$, then $b \in K$. Take an arbitrary $c \in C$ with $c \leq d$. Then $a \vee c \preceq b \vee c$ in $C^{\prime} \subset K$ and therefore $a \vee d=a \vee(c \vee d)=(a \vee c) \vee d \preceq(b \vee c) \vee d=b \vee d$

$K$ is simple, consequently, if two distinct elements of $C$ in $\bar{L}$ are congruent under a congruence $\Theta$, then $\Theta=\iota$ (the unit congruence). If we have a covering pair $a \prec b$ in $L$, then $b / a$ is prime-projective to a prime quotient of $C$ (see the strengthened version of the Jordan-Hölder theorem, for example, see J. B. Nation [6], Theorem 9.7), which proves that $\bar{L}$ is simple. Conditions (1) and (3) are obviously satisfied.

\section{REFERENCES}

[1] G. Czédli, E. T. Schmidt, Cover-preserving embedding of finite semimodular lattices into geometric lattices, manuscript, http://www.math.bme.hu/ schmidt/papers/112.pdf.

[2] G. Grätzer, D. Kelly, On congruence lattices of m-complete lattices Algebra Universalis 53 (2005), 253-265.

[3] G. Grätzer, E. W. Kiss, A construction of semimodular lattices, Order 2 (1986), 351-365.

[4] G. Grätzer, H. Lakser, On congruence lattices of m-complete lattices J. Austral Math. Soc. Ser. A 52 (1992), 57-87.

[5] G. Grätzer, T. Wares, Cover-preserving embeddings of finite semimodular lattices into simple semimodular lattices, manuscript, http://www.math.umanitoba.ca/homepages/gratzer.html/matharticles/smtosimple.pdf.

[6] J. B. Nation, Notes on Lattice Theory, unpublished Lecture Notes, http://www.math.hawaii.edu/ jb/books.html.

Mathematical Institute of the Budapest University of Technology and Economics, Múegyetem RkP. 3, H-1521 Budapest, Hungary

E-mail address, E. T. Schmidt: schmidt@math.bme.hu

$U R L:$ http://www.math. bme.hu/ schmidt/ 